\title{
ANISOTROPY OF THE EXCHANGE INTERACTION IN Mn-ALLOYED $\mathrm{II}_{3}-\mathrm{V}_{2}$ COMPOUNDS
}

\author{
W. LUBCZYŃSKI, J. CISOWSKI \\ Department of Solid State Physics, Polish Academy of Sciences \\ 41-800 Zabrze, ul. Kawalca 3, Poland
}

AND J.C. PORTAL

CNRS-SNCI, BP 166X, 38042 Grenoble Cedex, France

(Received)

\begin{abstract}
An anisotropy of the electron effective $g$-factor in highly degenerated tetragonal semimagnetic semiconductors (SMSC) alloyed with manganese has been found by us and by other workers when studying the Shubnikov-de Haas (SdH) effect for different orientations of the magnetic field with respect to the crystal axes. The detailed analysis of the experimental data by using a model based on an infinite Hamiltonian matrix, provides evidence for the anisotropy of the $p-d$ exchange interaction in the materials of interest.
\end{abstract}

\section{PACS numbers: 72.20.My}

The very complex crystal structure of $\mathrm{II}_{3}-\mathrm{V}_{2}$ semiconducting compounds can be approximated by a cubic structure with an additional tetragonal distortion. The presence of this axial distortion, which can be described by the crystal field splitting parameter $\delta$, leads to a strong anisotropy of the conduction band, as confirmed experimentally for $\mathrm{Cd}_{3} \mathrm{As}_{2}$ [1].

In the case of $\mathrm{Mn}$-alloyed $\mathrm{II}_{3}-\mathrm{V}_{2}$ SMSC with the tetragonal crystal structure, instead of a single constant $\beta$, as in cubic SMSC, describing the exchange interaction of $p$-like electrons with magnetic moments of $\mathrm{Mn}$ ions, one deals, in general, with two such constants, i.e. $\beta_{\perp}$ and $\beta_{\|}$[2]. This fact can additionally modify the anisotropy of the effective $g$-factor of free electrons in such materials.

The anisotropy of the conduction band in $\mathrm{Cd}_{3-x} \mathrm{Mn}_{x} \mathrm{As}_{2}$ (CMA) was studied for the first time by Blom et al. [3] who performed measurements of the SdH effect in monocrystalline samples for various orientations of the magnetic field 


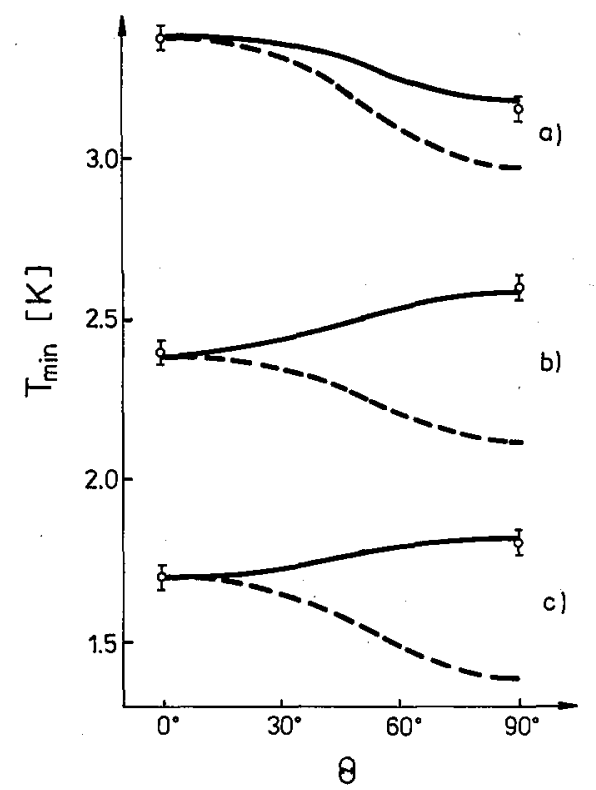

Fig. 1. Angular dependences of the first harmonic minimum position corresponding to $\nu=1.5(\mathrm{a})$ and $\nu=2.5(\mathrm{~b}, \mathrm{c})$, at constant magnetic field $B=1.65 \mathrm{~T}$ for $\mathrm{Cd}_{3-x} \mathrm{Mn}_{x} \mathrm{As}_{2}$. The experimental points are taken from Ref. [3]: $x=0.015$, the electron concentration $n=5.2 \times 10^{18} \mathrm{~cm}^{-3}$ (a); $x=0.03, n=5.3 \times 10^{18} \mathrm{~cm}^{-3}$ (b) $; x=0.03, n=3.5 \times 10^{18} \mathrm{~cm}^{-3}$ (c). The solid lines represent theoretical dependences for $\alpha=-0.4 \mathrm{eV}$ and the following sets of $\beta_{\perp}$ and $\beta_{\|}: \beta_{\perp}=2.08 \mathrm{eV}$ and $\beta_{\|}=2.20 \mathrm{eV}(\mathrm{a}), \beta_{\perp}=2.00 \mathrm{eV}$ and $\beta_{\|}=2.28 \mathrm{eV}$ (b, c). The dashed lines has been obtained for $\alpha=-0.4 \mathrm{eV}$ and $\beta_{\perp}=\beta_{\|}=\beta=2.08$ $\mathrm{eV}(\mathrm{a})$ or $\beta=2.00 \mathrm{eV}(\mathrm{b}, \mathrm{c})$.

with respect to the crystal axes. Anisotropy of the effective $g$-factor obtained in such a way turned out, however, to be not so strong as predicted by Neve et al. [4], and less pronounced than in $\mathrm{Cd}_{3} \mathrm{As}_{2}$. A similarly weak angular dependence of the $g$-factor has been observed by us in $\mathrm{Cd}_{3-x-y} \mathrm{Mn}_{x} \mathrm{Zn}_{y} \mathrm{As}_{2}$ (CZMA) [5].

In order to describe the experimental data, we have used a theoretical approach going beyond that taken in the earlier works and basing on an infinite Hamiltonian matrix found for the case of an arbitrary direction of the magnetic field. The details of the calculations are presented in [5].

The amplitude of the first harmonic of the $\mathrm{SdH}$ oscillations is modulated by the factor $\cos (\pi \nu)$, where $\nu$ is the ratio between the spin- and Landau splittings $\left(\nu=g^{*} m^{*} / 2 m_{0}\right.$, where $g^{*}$ is the electron effective $g$-factor, $m^{*}$ is the effective mass and $m_{0}$ denotes the free electron mass). For SMSC, the effective $g$-factor is a function of temperature $T$ and magnetic field $B$ [2] and the zeros (or minima, if the spin-dependent scattering is operating [6]) of the first harmonic amplitude can be observed, whenever $B$ or $T$ are such that $\nu=n+1 / 2$ with $n$ being an integer. For example, keeping $B$ constant, one can measure the angular dependence of the 
first harmonic minimum position as a function of $T$ and vice versa.

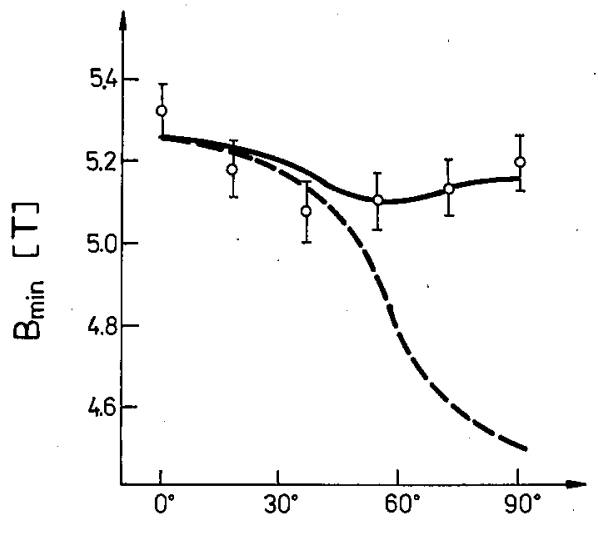

$\Theta$

Fig. 2. Angular dependence of the first harmonic minimum position corresponding to $\nu=0.5$, at constant temperature $T=4.2 \mathrm{~K}$ for $\mathrm{Cd}_{3-x-y} \mathrm{Mn}_{x} \mathrm{Zn}_{y} \mathrm{As}_{2}$ with $x=0.015$, $y=0.4$ and $n=2.35 \times 10^{18} \mathrm{~cm}^{-3}$. The solid line represents theoretical dependence for $\alpha=-0.4 \mathrm{eV}, \beta_{\perp}=1.82 \mathrm{eV}$ and $\beta_{\|}=2.04 \mathrm{eV}$. The dashed line has been obtained for $\alpha=-0.4 \mathrm{eV}$ and $\beta_{\perp}=\beta_{\|}=\beta=1.82 \mathrm{eV}$.

Comparison between the anisotropy of the first harmonic minimum positions $T_{\min }$ found in such a way for CMA [3] and that obtained from our theoretical model are presented in Fig. 1 (only the data for $\theta=0^{\circ}$ and $90^{\circ}$ were available; where $\theta$ denotes the angle between the magnetic field direction and tetragonal axis). An analogous comparison for CZMA measured by us at constant temperature is presented in Fig. 2. Values of the crystal field splitting $\delta$ have been estimated from the angular dependences of the period of SdH oscillations [5]. On the other hand, values of energy gap have been calculated from the linear dependence on composition [7, 8]. Other band structure parameters have been taken as for $\mathrm{Cd}_{3} \mathrm{As}_{2}$ [1] and the thermal average spin per Mn site - from independent magnetization measurements [9, 10]. The dashed and solid lines in Figs. 1 and 2 have been calculated for two different cases as far as the problem of anisotropy of the exchange interaction is concerned. In the first attempt, we have tried to describe the angular dependence of $B_{\min }$ (or $T_{\min }$ ) by an isotropic exchange interaction putting $\beta_{\perp}=$ $\beta_{\|}=\beta$ (dashed lines). It can be seen that the band structure anisotropy alone $(\delta \neq 0)$ is unable to describe the experimental results. Therefore, we have allowed for an anisotropy between $\beta_{\perp}$ and $\beta_{\|}$, what drastically changes the character of the angular dependences of $B_{\min }$ or $T_{\min }$ (solid lines) and gives a very good agreement with the experimental data.

In conclusion, our calculations based on the band structure model of a tetragonal SMSC in a quantising magnetic field give a strong evidence that the $p-d$ exchange interaction is anisotropic in the investigated materials. 


\section{References}

[1] F.A.P. Blom, in Narrow Gap Semiconductors Physics and Applications in series Lecture Notes in Physics, Vol. 133, Ed. W. Zawadzki, Springer-Verlag, New York 1980 , p. 191.

[2] J. Kossut, in Semiconductors and Semimetals, Vol 25, Eds. J.K. Furdyna, J. Kossut, Academic Press, Boston 1988, p. 183.

[3] F.A.P. Blom, J.J. Neve, P.A.M. Nouvens, in Proc. 16th Int. Conf. Phys. Semicond., Montpellier 1982, Ed. M. Averous, North-Holland, Amsterdam 1983, p. 470.

[4] J.J. Neve, J. Kossut, C.M. van Es, F.A.P. Blom, J. Phys. C 15, 4895 (1982).

[5]. W. Lubczyński, J. Cisowski, J. Kossut, J.C. Portal, Solid State Commun. 77, 541 (1991).

[6] W. Lubczyński, J. Cisowski, J. Kossut, J.C. Portal, Semicond. Science and Technol., in press.

[7] H.M.A. Schleijpen, Ph.D. thesis, Eindhoven, The Netherlands 1987, unpublished.

[8] W. Lubczyński, J. Cisowski, J.C. Portal, Phys. Status Solidi A 120, 525 (1990).

[9] W. Lubczyński, J. Cisowski, H. Bednarski, J. Voiron, J.C. Portal, J.C. Picoche, Acta Phys. Polon. A77, 175 (1990).

[10] C.J.M. Denissen, H. Nishihara, J.C. van Gool, W.J.M. de Jonge, Phys. Rev. $B$ 33, 7637 (1986). 